BASIC RESEARCH

\title{
Atorvastatin inhibits calcification and enhances nitric oxide synthase production in the hypercholesterolaemic aortic valve
}

\author{
N M Rajamannan, M Subramaniam, S R Stock, N J Stone, M Springett, K I lgnatiev, \\ J P McConnell, R J Singh, R O Bonow, T C Spelsberg
}

Heart 2005;91:806-810. doi: 10.1136/hrt.2003.029785

See end of article for authors' affiliations

Correspondence to: Dr Nalini M Rajamannan Northwestern University Feinberg School of Medicine, 303 E Chicago Avenue, Tarry 12-703, Chicago, IL 60611, USA; n-rajamannan@ northwestern.edu

Accepted 18 August 2004

\begin{abstract}
Objective: To study in a rabbit model the expression of endothelial nitric oxide synthase (eNOS) in association with the development of calcification of the aortic valve, and to assess the effects of atorvastatin on eNOS expression, nitrite concentration, and aortic valve calcification.

Methods: Rabbits ( $\mathrm{n}=48$ ) were treated for three months: 16, forming a control group, were fed a normal diet; 16 were fed a $0.5 \%$ (wt/wt) high cholesterol diet; and 16 were fed a $0.5 \%$ (wt/wt) cholesterol diet plus atorvastatin $(2.5 \mathrm{mg} / \mathrm{kg} /$ day). The aortic valves were examined with eNOS immunostains and western blotting. Cholesterol and high sensitivity $\mathrm{C}$ reactive protein (hsCRP) concentrations were determined by standard assays. Serum nitrite concentrations were measured with a nitric oxide analyser. eNOS was localised by electron microscopy and immunogold labelling. Calcification in the aortic valve was evaluated by micro-computed tomography (CT).

Results: Cholesterol, hsCRP, and aortic valve calcification were increased in the cholesterol fed compared with control animals. Atorvastatin inhibited calcification in the aortic valve as assessed by micro-CT. eNOS protein concentrations were unchanged in the control and cholesterol groups but increased in the atorvastatin treated group. Serum nitrite concentrations were decreased in the hypercholesterolaemic animals and increased in the group treated with atorvastatin.

Conclusion: These data provide evidence that chronic experimental hypercholesterolaemia produces bone mineralisation in the aortic valve, which is inhibited by atorvastatin.
\end{abstract}

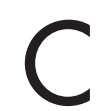
alcific aortic valve disease is the most common indication for cardiac valve replacement in developed countries. Calcific aortic stenosis shares common risk factors with vascular atherosclerosis: hypercholesterolaemia, smoking, hypertension, and male sex. ${ }^{1}$ The cellular mechanisms for this disease are emerging. We have recently shown that experimental hypercholesterolaemia produces an atherosclerotic lesion in the aortic valve, which contains cells expressing early osteoblast marker genes similar to those markers found in skeletal bone formation. ${ }^{2}$ We and others have also shown that these osteoblast markers and osteoblast bone formation are present in human calcified aortic valves. $^{3}{ }^{4}$ The intermediate signalling steps between lipid accumulation and calcification have not been clearly established in the aortic valve as they have been in the aorta and the aortic arch. ${ }^{56}$

Structurally, an endothelial layer lines the aortic valve leaflet. We proposed that endothelial nitric oxide synthase (eNOS) produced in the valve endothelium has a role in the physiological cellular regulation of this tissue similar to the role of eNOS in vascular endothelium, which has not been reported before this study. Nitric oxide is generated in vascular endothelial cells by eNOS and is responsible for endothelial dependent vasorelaxation, inhibition of smooth muscle cell proliferation, and decreased synthesis of extracellular matrix proteins. ${ }^{7}$ In the presence of oxidised low density lipoprotein, eNOS is downregulated by destabilising eNOS mRNA. ${ }^{8}$ Hydroxymethyl glutaryl coenzyme A (HMG$\mathrm{CoA}$ ) reductase inhibitors may reverse this downregulation during hypercholesterolaemic conditions by stabilising eNOS mRNA. ${ }^{9}$
In this study, we hypothesised that eNOS is downregulated in the setting of chronic hypercholesterolaemia and that the resulting decrease in nitrite production contributes to calcification of the aortic valve. We also assessed the effects of atorvastatin on eNOS expression, nitrite concentrations, and calcification of the aortic valve.

\section{MATERIAL AND METHODS}

Animals and valve specimens

Male New Zealand white rabbits weighing $2.5-3.0 \mathrm{~kg}$ were assigned to a control group $(n=16)$, a $0.5 \%$ cholesterol fed group ( $\mathrm{n}=16)$, or a cholesterol fed and atorvastatin treated group $(\mathrm{n}=16)$. All animals were fed ad libitum for three months. Control rabbits were fed a standard diet. Cholesterol fed animals received a diet supplemented with $0.5 \%$ (wt/wt) cholesterol (Purina Mills, Woodmont, Indiana, USA), and the cholesterol fed and atorvastatin treated group were given atorvastatin $2.5 \mathrm{mg} / \mathrm{kg}$ daily. Following this three months period, the rabbits were anaesthetised with intramuscular ketamine/xylazine $(40 / 5 \mathrm{mg} / \mathrm{kg})$ and then were euthanised with intracardiac administration of $1 \mathrm{ml}$ of pentobarbital/ phenytoin (Beuthanasia; Schering Plough Animal Health). All experiments were performed in an animal facility accredited by the Association for Assessment and Accreditation of Laboratory Animal Care, Inc (ACUC-A3283-01, 1-08382). Immediately after excision from the heart, one leaflet from each aortic valve was fixed in $4 \%$ buffered formalin for

Abbreviations: $\mathrm{CT}$, computed tomography; eNOS, endothelial nitric oxide synthase, HMG-CoA, hydroxymethyl glutaryl coenzyme A; hsCRP, high sensitivity $C$ reactive protein 
24 hours and then embedded in paraffin. Paraffin embedded sections $(6 \mu \mathrm{m})$ were cut for immunostaining.

\section{Lipid concentrations, high sensitivity $\mathrm{C}$ reactive protein, and nitric oxide measurements}

Blood samples were centrifuged at $2000 \mathrm{rpm}$ for 10 minutes at $4^{\circ} \mathrm{C}$ and the serum was stored at $-70^{\circ} \mathrm{C}$. Total serum cholesterol concentrations were measured by standard enzymatic techniques. The high sensitivity $\mathrm{C}$ reactive protein (hsCRP) concentrations in the serum were measured by an automated latex particle enhanced immunoturbidometric method from Kamiya (Seattle, Washington, USA). Serum samples $(100 \mu \mathrm{l})$ containing $\mathrm{NO}_{2}$ were refluxed in acetic acid/ $\mathrm{NaI}$ and $\mathrm{NO}_{2}$. Under these conditions, $\mathrm{NO}_{2}$ was quantitatively reduced to nitric oxide, which was quantified by a chemiluminescence detector after each reaction with ozone. Net $\mathrm{NO}_{2} /$ $\mathrm{mg}$ of protein was calculated from each experiment (NOA, Sievers Instruments, Boulder, Colorado, USA).

\section{Immunohistochemistry}

The paraffin embedded aortic valve leaflets were immunostained for eNOS to confirm the presence of this protein in the aortic valves. After fixation, slides were treated sequentially with $3.0 \% \mathrm{H}_{2} \mathrm{O}_{2}$ for 15 minutes and normal rabbit serum for 20 minutes, washed with phosphate buffered saline (wt/wt) for 10 minutes, and incubated in 1:25 eNOS antibody (BD Pharmagen, Chicago, Illinois, USA) for 16 hours at $4^{\circ} \mathrm{C}$. To develop colour, slides were incubated in 3-amino-9-ethylcarbazole (Sigma Chemical Co, St Louis, Missouri, USA) and washed with water for five minutes.

\section{Western blotting}

Aortic valves from each of the treatment groups were obtained at the time of death and tested for selected proteins by western blot analysis. Nitrocellulose membranes (Amersham, Biosciences, Piscataway, New Jersey, USA) were blocked in TBS-T (20 mM Tris base (pH 7.6), $137 \mathrm{mM} \mathrm{NaCl}$, $0.1 \%$ Tween-20) supplemented with 5\% (wt/vol) bovine serum albumin for one hour at room temperature. After blocking, blots were treated with the primary antibody eNOS (Signal Transduction Laboratories, Cell Signalling Technology, Beverly, Massachusetts, USA) for one hour at room temperature and a control antibody $\alpha$ actin (Sigma). Blots were washed several times with TBS-T after the incubation with the primary and secondary antibodies. The secondary antibody was a horseradish peroxidase conjugated to a mouse antibody at a 1:7500 dilution in TBS-T supplemented with 5\% bovine serum albumin. The blots were treated with the secondary antibody for one hour at room temperature.

The enhanced chemiluminescent method (Amersham Life Sciences) was used to optimise visualisation and quantification of the western blots. Appropriate bands from autoradiographs are quantified by laser scanning densitometry (NIH Image, www.nih.gov). When needed, the blots were stripped by incubation for 30 minutes at $50^{\circ} \mathrm{C}$ in stripping buffer (62.5 mM Tris-HCl (pH 6.5), $100 \mathrm{mM} \mathrm{2-ME,} \mathrm{2 \%} \mathrm{vol/vol}$ sodium dodecyl sulfate), washed twice with TBS-T, and blocked with TBS-T supplemented with 5\% bovine serum albumin.

\section{Immunogold electron microscopy}

The valve leaflets were fixed in $4 \%$ formaldehyde plus $0.2 \%$ glutaraldehyde in phosphate buffer overnight, rinsed in phosphate buffer, partially dehydrated to $80 \%$ ethanol, and embedded in LR White resin. Sections were then incubated in mouse monoclonal antibody to eNOS diluted to 1:250. Sections were examined after staining with uranyl acetate and lead citrate as previously described. ${ }^{10}$

\section{Micro-computed tomography}

After they were fixed in formalin, aortic valves and aortas were examined with a Scanco Micro-CT-40 system operated at $45 \mathrm{kV}$ to assess the presence and extent of calcification. Sampling was with $\sim 8 \mu \mathrm{m}$ voxels (volume elements) and maximum sensitivity (1000 projections, 2048 samples, and $0.3 \mathrm{~s} /$ projection integration).

\section{Statistical analysis}

The three groups were compared by analysis of variance. The Scheffe method of adjustment was used to make multiple pairwise comparisons. All statistical tests were two tailed and $\mathrm{p}<0.05$ was considered significant.

\section{RESULTS}

\section{Light microscopy and immunostaining}

The normal aortic valve surface from control animals appeared thin and intact, with a smooth endothelial cell layer covering the entire surface and a thin collagen layer within the spongiosa. There was little eNOS immunostaining in the endothelium (fig 1Al, fig 1B1). In contrast, the aortic valves from the hypercholesterolaemic animals had an atherosclerotic fatty streak lesion along the aortic surface that was identifiable by light microscopy (fig 1A2, fig 1B2) with minor evidence of eNOS immunostaining along the surface of the aortic valve. The amount of atherosclerotic plaque burden decreased greatly in the atorvastatin treated rabbits, with a positive immunostain for eNOS in endothelial layer of the aortic valve (fig 1A3). Higher magnification of the endothelial lining of the aortic valve showed eNOS immunostaining in the atorvastatin treated aortic valves (fig 1B3).

\section{Immunogold electron microscopy for eNOS expression and mineralisation}

The presence of eNOS and evidence of mineralisation were assessed at the ultrastructural level in the three groups. In the control group, the eNOS label was localised to the endothelial cells (fig 1C1, fig 1D1). In fig $1 \mathrm{C} 2$ there are areas of mineralisation (see arrow). In the hypercholesterolaemic valves, there was a major accumulation of lipids with a small amount of eNOS expression (fig 1D2), which is seen in the higher magnification electron microscopy scans. The atorvastatin treated aortic valves (fig 1C3, fig 1D3) appeared similar to the control aortic valves with eNOS expression near the endothelium and less lipid infiltration.

\section{Micro-computed tomography}

Micro-computed tomography (CT) was used to evaluate the development of calcification in valve leaflets and the aorta in each of the treatment groups. Control aortic valves and aortas had little to no mineralisation (fig $2 \mathrm{~A}$ ). The hypercholesterolaemic aortic valves and aortas appeared to be in early stages of calcification (figure 2B). In the group given both cholesterol and atorvastatin the aortic valves and aortas could not be distinguished from those of the controls (fig 2C).

\section{Western blot}

Analysis of eNOS protein expression of the aortic valves indicated that eNOS expression in the control and cholesterol treatments were not substantially different. However, protein expression increased 150-fold in the atorvastatin treated aortic valve over the control and the cholesterol aortic valves (fig 1E). Table 1 lists results of quantification of eNOS by western blotting.

Serum cholesterol, hsCRP, and nitrite concentrations Table 1 lists the relative serum concentrations in each of the three treatment arms. Total plasma cholesterol concentrations were significantly higher in the cholesterol fed than in 


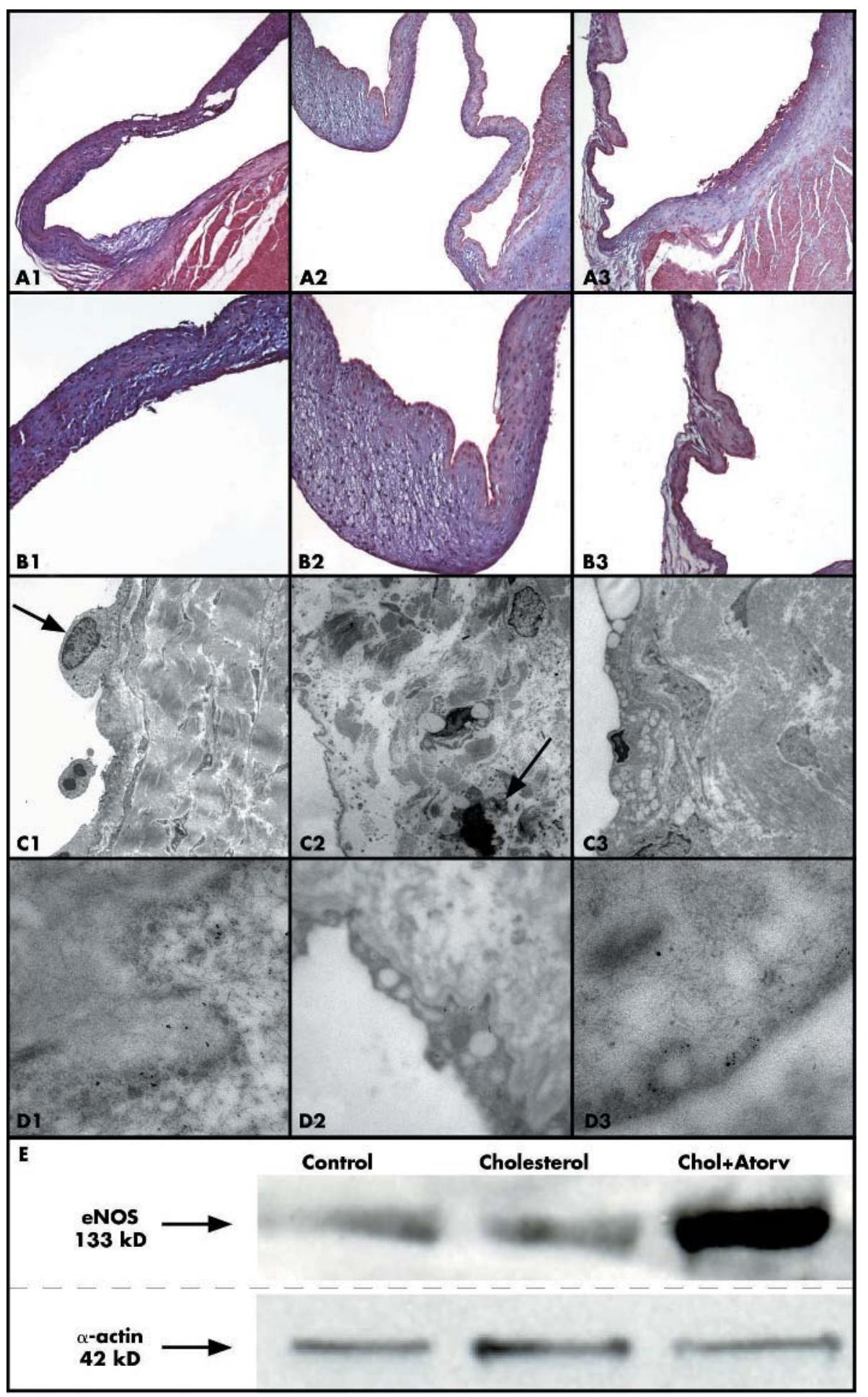

Figure 1 Evidence for endothelial nitric oxide synthase (eNOS) localisation and calcification in the aortic valve. Left column, control diet; middle column, cholesterol (Chol) diet; right column, cholesterol diet plus atorvastatin (Atorv). In each panel, the aortic valve leaflet is positioned on the left, with the aorta on the right. (A) eNOS immunostaining (all frames $4 \times$ magnification). (B) eNOS immunostaining (all frames $20 x$ magnification). (C) eNOS immunogold electron microscopy (low magnification, $25000 \times$ ) with areas of mineralisation (arrows). (D) eNOS immunogold electron microscopy (high magnification, $125000 \times$ ). (E) Western blot analysis of eNOS protein expression and the control.

control animals (44.7 (16.5) $v \quad 1.17 \quad(0.15) \mathrm{mmol} / \mathrm{l}$, $\mathrm{p}<0.001)$. Atorvastatin treated rabbits manifested significantly lower cholesterol concentrations $(28.2$ (13) mmol/l, $\mathrm{p}<0.001)$ than the rabbits receiving the cholesterol diet alone. Concentrations of hsCRP were significantly higher in the cholesterol fed than in control rabbits (4.1 (1.9) $v 0.3$ (0.05) $\mathrm{mg} / \mathrm{l}, \mathrm{p}<0.001)$. hsCRP concentrations were near normal after cholesterol plus atorvastatin (0.4l (0.4) $\mathrm{mg} / \mathrm{l})$. Serum nitrite concentrations were significantly lower in the hypercholesterolaemic than in the control (198.2 (108.8) $v$
$994.7(392.5) \mathrm{nmol} / \mathrm{l}, \mathrm{p}<0.005)$ and atorvastatin treated rabbits $(745.6(340.8) \mathrm{nmol} / \mathrm{l}, \mathrm{p}<0.005)$.

\section{DISCUSSION}

Calcification of aortic valves has been described in the literature for over 100 years. Until recently, only descriptive studies delineated this process. In our previous paper we reported that during two months of experimental hypercholesterolaemia the aortic valve develops an atherosclerotic lesion, which expresses extracellular osteoblast-type markers 


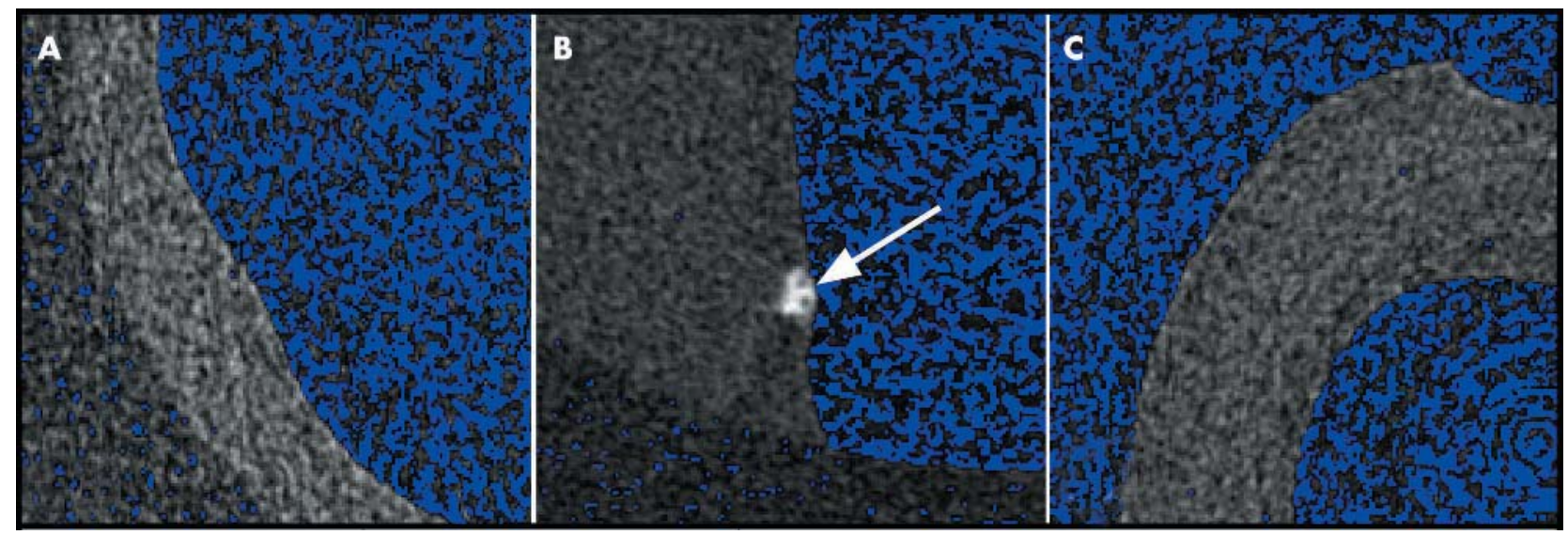

Figure 2 Micro-computed tomography of the aortic valves in the treatment groups indicating an area of calcification in the cholesterol treated aortic valve. Aortic valves and aortas from the (A) control group; (B) hypercholesterolaemic group (the arrow shows early stages of calcification); and (C) hypercholesterolaemic, atorvastatin treated group.

important in skeletal bone calcification. ${ }^{2}$ However, the first study did not find evidence of calcification with two months of cholesterol treatments. The current study found calcification and abnormal eNOS concentrations in the aortic valve after three months of cholesterol feeding. Feron and colleagues ${ }^{11}$ have previously shown that eNOS concentrations are decreased with hypercholesterolaemia. Myers and colleagues $^{12}$ have further shown that decreased nitric oxide concentrations are associated with an increase in collagen matrix production within the vasculature. These experiments in the vasculature are important studies supporting the hypothesis tested in this current study. We have already shown in our previously published two month cholesterol model $^{2}$ that cholesterol increases the synthesis of bone matrix proteins in the aortic valve. This current study tested three months of cholesterol treatment to determine whether mineralisation begins to develop in the aortic valve at the micro-CT level. These studies provide the first evidence that calcification develops in the hypercholesterolaemic aortic valve by Micro-CT analysis with an associated decrease in eNOS protein concentrations.

In this study we also studied markers of oxidative stress (serum nitrites) and inflammation (hsCRP). Increased CRP concentration is an inflammatory marker associated with high cholesterol treatments as reported by Galante and colleagues. ${ }^{13}$ Our data indicate that increased CRP is associated with experimental aortic valve calcification in the high cholesterol treated aortic valves. In this study, we also tested a marker of oxidative stress, serum nitrites. Lubrano and colleagues ${ }^{8}$ have further shown that increased lipoproteins decrease the serum nitrite concentrations in patients with familial hypercholesterolaemia. In this study, we evaluated serum nitrite concentrations in the rabbits as a

Table 1 Results of analyses of rabbit serum and aortic valves

\begin{tabular}{llll}
\hline Variable & Control group & $\begin{array}{l}\text { Cholesterol } \\
\text { group }\end{array}$ & $\begin{array}{l}\text { Cholesterol + } \\
\text { atorvastatin } \\
\text { group }\end{array}$ \\
\hline Cholesterol (mmol/l) & $1.17(0.15)$ & $44.7(16.5)$ & $28.2(13)^{*}$ \\
hsCRP (mg/l) & $0.3(0.05)$ & $4.1(1.9)$ & $0.41(0.4)^{*}$ \\
Nitrite (nmol/l) & $994.7(392.5)$ & $198.2(108.8)$ & $745.6(340.8)^{*}$ \\
eNOS (\% of control) & $101.9 \%$ & $85.2 \%$ & $248.8 \%$ \\
\hline
\end{tabular}

${ }^{*} \mathrm{p}<0.001$

eNOS, endothelial nitric oxide synthase; hsCRP, high sensitivity C reactive protein. marker of oxidative stress in the treatment groups. Serum nitrite concentrations decreased in the cholesterol treated rabbits and improved in the atorvastatin treated rabbits, which implies a reduction in oxidative stress, another pleiotrophic effect of statins. ${ }^{14}$

An important comparison was published by Drolet and colleagues, ${ }^{15}$ who showed in a rabbit model that experimental hypercholesterolaemia in the presence of vitamin D treatment produced an aortic valve lesion that is stenotic. Even though they have established a model of valve stenosis with these two treatments, their model may not be completely representative of the biological processes in human valve disease. Recently, Shioi and colleagues ${ }^{16}$ showed that vitamin $\mathrm{D}$ and cholesterol treated aortas develop a cartilaginous calcification. This type of bone formation occurs secondary to the chondrocyte differentiation pathway, which differs from the osteoblast differentiation pathway. These results may have implications in the modalities used to treat cardiovascular calcification in the future. Therefore, ongoing experimental studies are necessary with both models to define the types of bone formation and to compare these results with bone formation found in human aortic valve calcification to develop future medical treatments for this disease.

In conclusion, these data show that atorvastatin increases the protein expression and functional activity of eNOS, improves CRP concentrations, increases nitrite concentrations, and decreases calcification in rabbit aortic valves. These changes coincide with inhibition of calcification in the aortic valve and with inhibition of important bone matrix markers critical in osteoblast differentiation. ${ }^{2}$ Previously, we have shown that osteoblast differentiation is an important finding in human calcified valves removed at the time of surgical valve replacement. ${ }^{3}$ Therefore, targeting this cellular differentiation pathway is important in the understanding of the potential treatment of this disease. Recent retrospective clinical studies showed that treatment with HMG-CoA reductase inhibitors of patients with calcific aortic stenosis may slow the rate of calcification as shown by electron beam CT. ${ }^{17}$ These findings suggest that such treatment may, in patients in the early stages of this disease process, have a potential role in slowing the progression of aortic valve disease.

\section{ACKNOWLEDGEMENTS}

$\mathrm{N}$ R is supported by the US National Institutes of Health (1K08HL073927-01) and an American Heart Association Grant in Aid (0350564Z). The authors thank Frank Caira, Jason Gocek, and Trisha Smith for their technical support. 


\section{Authors' affiliations}

N M Rajamannan, N J Stone, R O Bonow, Division of Cardiology, Department of Medicine, Northwestern University Feinberg School of Medicine, Chicago, Illinois, USA

M Subramaniam, T C Spelsberg, Department of Biochemistry and Molecular Biology, Mayo Clinic, Rochester, Minnesota, USA

J P McConnell, R J Singh, Department of Laboratory Medicine and Pathology, Mayo Clinic

M Springett, Electron Microscopy Core Facility, Mayo Clinic S R Stock, K I Ignatiev, Institute for Bioengineering and Nanoscience in Advanced Medicine, Northwestern University Feinberg School of Medicine, Chicago, Illinois, USA

\section{REFERENCES}

1 Stewart BF, Siscovick D, Lind BK, et al. Clinical factors associated with calcific aortic valve disease. Cardiovascular health study. J Am Coll Cardiol 1997:29:630-4

2 Rajamannan NM, Subramaniam M, Springett $M$, et al. Atorvastatin inhibits hypercholesterolemia-induced cellular proliferation and bone matrix production in the rabbit aortic valve. Circulation 2002;105:2260-5.

3 Rajamannan NM, Subramaniam M, Rickard D, et al. Human aortic valve calcification is associated with an osteoblast phenotype. Circulation 2003; 107:2181-4.

4 Mohler ER 3rd, Gannon F, Reynolds C, et al. one formation and inflammation in cardiac valves. Circulation 2001;103:1522-8.

5 Van de Poll SW, Delsing DJ, Jukema JW, et al. Raman spectroscopic investigation of atorvastatin, amlodipine, and both on atherosclerotic plaque development in $\mathrm{APOE}^{\star} 3$ Leiden transgenic mice. Atherosclerosis 2002; 164:65-71.
6 Van De Poll SW, Romer TJ, Volger OL, et al. Raman spectroscopic evaluation of the effects of diet and lipid-lowering therapy on atherosclerotic plaque development in mice. Arterioscler Thromb Vasc Biol 2001;21:1630-5.

7 Wilcox JN, Subramanian RR, Sundell CL, et al. Expression of multiple isoforms of nitric oxide synthase in normal and atherosclerotic vessels. Arterioscler Thromb Vasc Biol 1997; 17:2479-88.

8 Lubrano V, Vasalle C, Blandizzi C, et al. The effect of lipoproteins on endothelial nitric oxide synthase is modulated by lipoperoxides. Eur J Clin Invest 2003;33:117-25.

9 Laufs U, La Fata V, Plutzky J, et al. Upregulation of endothelial nitric oxide synthase by HMG CoA reductase inhibitors. Circulation 1998;97:1 129-35.

10 Rajamannan NM, Springett MJ, Pederson LG, et al. Localization of caveolin 1 in aortic valve endothelial cells using antigen retrieval. J Histochem Cytochem 2002; $50: 617-28$

11 Feron O, Dessy C, Moniotte S, et al. Hypercholesterolemia decreases nitric oxide production by promoting the interaction of caveolin and endothelial nitric oxide synthase. J Clin Invest 1999;103:897-905.

12 Myers PR, Tanner MA. Vascular endothelial cell regulation of extracellular matrix collagen: role of nitric oxide. Arterioscler Thromb Vasc Biol 1998; 18:717-22.

13 Galante A, Pietroiusti A, Vellini $M$, et al. C-reactive protein is increased in patients with degenerative aortic valvular stenosis. J Am Coll Cardiol 2001;38:1078-82.

14 Laufs U, Liao JK. Isoprenoid metabolism and the pleiotropic effects of statins. Curr Atheroscler Rep 2003:5:372-8.

15 Drolet MC, Arsenault $M$, Couet J. Experimental aortic valve stenosis in rabbits $J$ Am Coll Cardiol 2003;41:1211-7.

16 Shioi A, Kizu A, Jono S, et al. Inactivation of Vitamin D receptor suppresses development of atherosclerotic calcification in ApoE-deficient mice. Cardiovasc Pathol 2004;13(3S):S15.

17 Shavelle DM, Takasu J, Budoff MJ, et al. HMG CoA reductase inhibitor (statin) and aortic valve calcium [comment]. Lancet 2002;359:1125-6.

\section{IMAGES IN CARDIOLOGY}

\section{Left atrial membrane, left ventricular dysfunction, and mitral regurgitation in an adult}

eft atrial division may present in adults, often with an atrial septal defect (ASD), causing pulmonary hyperten- sion and breathlessness. A 28 year old man presented with a six month history of breathlessness and palpitations. Atrial fibrillation, pansystolic murmur, and bi-basal crepitations were noted. Transoesophageal echocardiogram (TOE) (panel A) showed a left ventricular ejection fraction of 32\%, severe central mitral regurgitation with normal leaflets, chordae and papillary muscles, and a left atrial dividing membrane with a small posteromedial communication. There was no ASD. Pulmonary artery pressure on Doppler ultrasound was $90 \mathrm{~mm} \mathrm{Hg}$.

A direct operative approach entered the superior chamber (with forceps through the communication) (panel B). Membrane excision and ring mitral valvoplasty were performed (TOE confirmed competence). The patient regained sinus rhythm and recovered well. TOE four months postsurgery showed an ejection fraction of $41 \%$ and a pulmonary artery pressure of $40 \mathrm{~mm} \mathrm{Hg}$.

Cor triatriatum sinistrum consists of a fibromuscular membrane dividing the left atrium into a proximal chamber receiving pulmonary veins and distal chamber leading to the left ventricle. There may be communications, and an ASD may vent the pulmonary circulation. Here there was a $0.5 \mathrm{~cm}$ transmembrane defect (panel B). Left ventricular failure and atrial fibrillation probably induced deterioration. Surgical intervention is the principal treatment with excellent postoperative results.
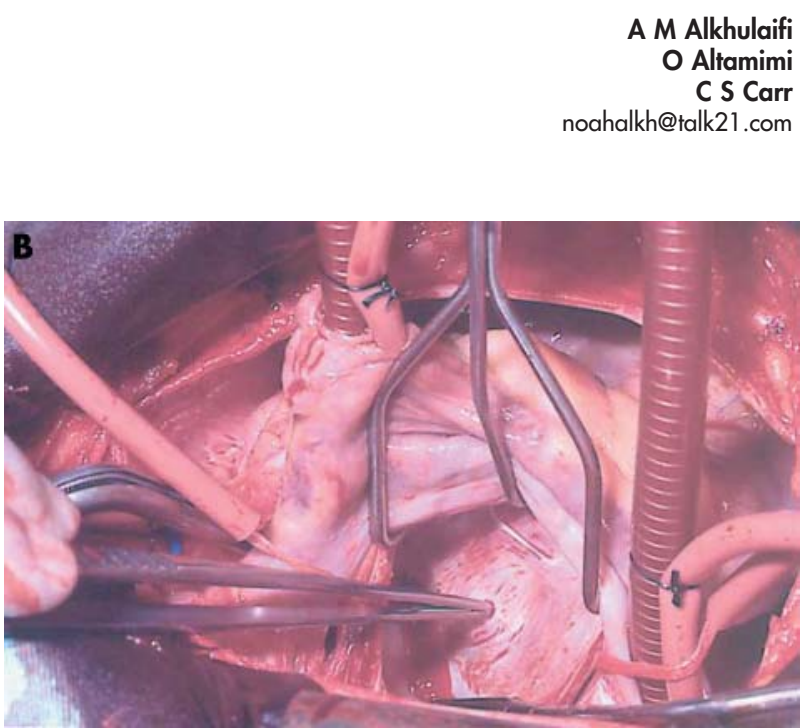

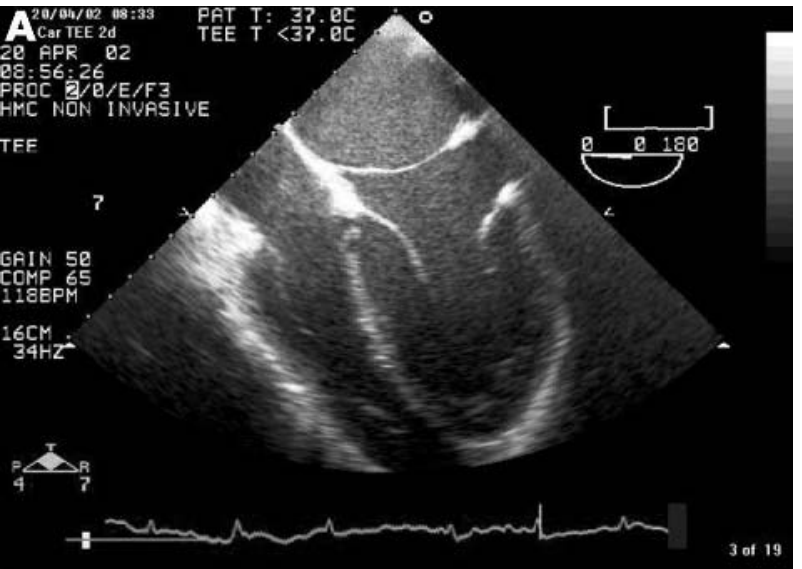

